\title{
Article
}

\section{Prognostic significance of early urinary catheterization after acute stroke: Secondary analyses of the international HeadPosT trial}

Ouyang, Menglu, Billot, Laurent, Song, Lili, Wang, Xia, Roffe, Christine, Arima, Hisatomi, Lavados, Pablo M., Hackett, Maree, Watkins, Caroline Leigh and Et, Al

Available at http://clok.uclan.ac.uk/31854/

Ouyang, Menglu, Billot, Laurent, Song, Lili, Wang, Xia, Roffe, Christine, Arima, Hisatomi, Lavados, Pablo M., Hackett, Maree ORCID: 0000-0003-1211-9087, Watkins, Caroline Leigh ORCID: 0000-0002-9403-3772 et al (2021) Prognostic significance of early urinary catheterization after acute stroke: Secondary analyses of the international HeadPoST trial. International Journal of Stroke, 16 (2). pp. 200-206. ISSN 1747-4930

It is advisable to refer to the publisher's version if you intend to cite from the work. http://dx.doi.org/10.1177/1747493020908140

For more information about UCLan's research in this area go to http://www.uclan.ac.uk/researchgroups/ and search for <name of research Group>.

For information about Research generally at UCLan please go to http://www.uclan.ac.uk/research/

All outputs in CLoK are protected by Intellectual Property Rights law, including Copyright law. Copyright, IPR and Moral Rights for the works on this site are retained by the individual authors and/or other copyright owners. Terms and conditions for use of this material are defined in the policies page. 


\section{Prognostic significance of early urinary catheterization after acute stroke: secondary}

analyses of the international HeadPoST trial

Menglu Ouyang MPH, ${ }^{1,2}$ Laurent Billot MRes, ${ }^{1}$ Lili Song MD PhD,${ }^{1,2}$ Xia Wang PhD, ${ }^{1}$ Christine Roffe, ${ }^{3}$ Hisatomi Arima MD PhD, ${ }^{1,4}$ Pablo M. Lavados MD MPH, ${ }^{5}$ Maree L. Hackett PhD, ${ }^{1,6}$ Verónica V. Olavarría MD MSc., ${ }^{5}$ Paula Muñoz-Venturelli MD PhD, ${ }^{1,7}$ Sandy Middleton PhD ${ }^{8}$ Octavio M. Pontes-Neto MD PhD, ${ }^{9}$ Tsong-Hai Lee MD PhD, ${ }^{10}$ Caroline L. Watkins PhD, ${ }^{6}$ Thompson Robinson MD, ${ }^{11}$ Craig S. Anderson MD PhD ${ }^{1,2,7,12,13}$

${ }^{1}$ The George Institute for Global Health, Faculty of Medicine, University of New South Wales, Sydney, Australia

${ }^{2}$ The George Institute China at Peking University Health Science Center, Beijing, China

${ }^{3}$ Department of Neurosciences, Royal Stoke University Hospital, UK

${ }^{4}$ Department of Preventive Medicine and Public Health, Faculty of Medicine, Fukuoka University, Fukuoka, Japan

${ }^{4}$ Department of Neurosciences, Royal Stoke University Hospital, UK

${ }^{5}$ Unidad de Neurología Vascular, Servicio de Neurología, Departmento de Neurologia and Psiquiatria, Clínica Alemana de Santiago

${ }^{6}$ Faculty of Health and Wellbeing, University of Central Lancashire, Preston, Lancashire, UK ${ }^{7}$ Universidad del Desarrollo, School of Medicine-Clínica Alemana, ICIM, Center for Clinical Studies, Santiago, Chile

${ }^{8}$ Nursing Research Institute, St Vincent's Health (Sydney) Australia, Australian Catholic University, Sydney, Australia

${ }^{9}$ Stroke Service - Neurology Division, Department of Neuroscience and Behavioral Sciences, Ribeirão Preto Medical School, University of Sao Paulo, Ribeirão Preto - SP, Brazil

${ }^{10}$ Stroke Center and Department of Neurology, Linkou Chang Gung Memorial Hospital and College of Medicine, Chang Gung University, Taoyuan, Taiwan

${ }^{11}$ Department of Cardiovascular Sciences and NIHR Leicester Biomedical Research Centre, University of Leicester, Leicester, UK

${ }^{12}$ Neurology Department, Royal Prince Alfred Hospital, Sydney Health Partners, Sydney, Australia

${ }^{13}$ Heart Health Research Center, Beijing, China

\section{Corresponding Author}

Professor Craig S Anderson

The George Institute for Global Health, PO Box M201, Missenden Rd., NSW 2050, Australia T: +61-2-9993-4500; F: +61-2-9993-4502; E: canderson@ georgeinstitute.org.au

Total Word Count: 4173 (Abstract 244; Main 2083) 
Brief title: Urinary catheterization and stroke outcome

Keywords: urinary catheter, disability, acute stroke, urinary tract infection, clinical trial

Tables: 3

Supplemental tables: 8

Supplemental figures: 4

Number of references: 20 


\begin{abstract}
Background: An indwelling urinary catheter (IUC) is often inserted to manage bladder dysfunction but its impact on prognosis is uncertain. We aimed to determine the association of IUC use on clinical outcomes after acute stroke in the international, multi-center, cluster crossover, Head Positioning in Acute Stroke Trial (HeadPoST).
\end{abstract}

Methods: Data were analyzed on HeadPoST participants $(n=11,093)$ randomly allocated to the lying-flat or sitting-up head position. Binomial, logistic regression, hierarchical mixed models were used to determine associations of early insertion of IUC within 7 days postrandomization and outcomes of death or disability (defined as 'poor outcome', scores 3-6 on the modified Rankin scale) and any urinary tract infection (UTI) at 90 days with adjustment of baseline and post-randomization management covariates.

Results: Overall, 1167 (12\%) patients had an IUC, but the frequency and duration of use varied widely across patients in different regions. IUC use was more frequent in older patients, and those with vascular comorbidity, greater initial neurological impairment (on the National Institutes of Health stroke scale), and intracerebral hemorrhage as the underlying stroke type. IUC use was independently associated with poor outcome (adjusted odds ratio [aOR] 1.40, 95\% confidence interval [CI] 1.13-1.74), but not with UTI after adjustment for antibiotic treatment and stroke severity at hospital separation (aOR 1.13, 95\% CI 0.59-2.18). The number exposed to IUC for poor outcome was 13.

Conclusions: IUC use is associated with a poor outcome after acute stroke. Further studies are required to inform appropriate use of IUC. 
Bladder dysfunction is common after acute stroke, affecting at least one third of patients. ${ }^{1} \mathrm{~A}$ urodynamic study suggested frequencies of urinary incontinence of $73 \%$ and $64 \%$, and urinary retention of $13 \%$ and $52 \%$, in patients with acute intracerebral hemorrhage (ICH) and ischemic stroke, respectively. ${ }^{2}$ An indwelling urine catheter (IUC) is often inserted to manage these conditions ${ }^{3}$ but the benefits may be offset by increased mortality and morbidity, especially in the context of incontinence. ${ }^{4,5}$ Guidelines $^{6,7}$ therefore recommend cautious use of IUC, and for their early removal to avoid urinary tract infection (UTI) and other sepsis. However, these recommendations are based on level $\mathrm{C}$ grade of evidence, since the data are derived from studies limited by small sample size, retrospective design, and incomplete adjustment for confounding. We aimed to determine associations of IUC use and 90-day clinical outcomes in stroke patients with a broad range of characteristics who participated in the international Head Positioning in Acute Stroke Trial (HeadPoST). ${ }^{8}$

\section{Methods}

\section{Study population}

HeadPoST was an international, multicenter, cluster crossover, clinical trial that involved 11,093 adults ( $\geq 18$ years) with acute stroke randomly allocated to the lying flat or sitting up head position soon at 114 hospitals in 9 countries from March 2015 to November $2016 .{ }^{8}$ A guardian consent process was used to implement the randomized intervention as a policy of usual service delivery to a pre-defined patient cluster; patients provided consent for use of their medical record data and centralized telephone follow-up. HeadPoST is registered at ClinicalTrials.gov (NCT02162017).

\section{Procedures}

Demographic, medical history and clinical information, included the severity of neurological impairment on the National Institutes of Health Stroke Scale (NIHSS), were collected at 
baseline. Data of IUC use, including insertion date, along with other pre-defined management interventions, repeat NIHSS and an assessment of functional status on the simplified modified Rankin scale (mRS), were collected at Day 7 post-randomization (or at hospital separation if earlier). Recorded IUC use was assumed as the first insertion after hospital admission and duration of use was censored at Day 7. Trained staff, blind to treatment allocation, contacted patients not known to have died by telephone to assess their functional status on the mRS at 90-days. The primary outcome for these analyses was death or dependency (mRS scores 3-6). The secondary outcome was UTI identified from details related to serious adverse events (SAEs) reported by site investigators to the end of follow-up at 90 days. $^{8}$

\section{Statistical analysis}

Binomial, logistic regression, hierarchical mixed models were used to adjust for the fixed effects of head position (lying-flat versus sitting-up) and cross-over period, random effects of cluster, and random interaction effects between cluster and crossover period. The term 'unadjusted' was used for convenience in defining this initial mixed model. Potential confounders at baseline and Day 7 post-randomization $(\mathrm{P}$ value $<0.2)$ in univariate analyses (Table 1; Supplemental Tables S1 and S2) defining associations of IUC and outcomes were included in sequential hierarchical logistic regression models with (model I) country groups according to region, (model II) baseline characteristics, and (model III) management covariates. As the NIHSS and mRS scores at Day 7 (or earlier) were correlated (Spearman's rank correlation=0.72), the former scale was used in model III to adjust for early neurological change. As there was a high proportion of missing Day $90 \mathrm{mRS}$ scores (12\%), multiple imputation was conducted for a sensitivity analysis with all covariates (include outcome variable) in the mixed model for analysis (method was described in Supplemental Material). Considering UTI is an intermediate variable for IUC and poor functional outcome, an 
additional sensitivity analysis was conducted to assess the strength of association in patients without UTI. Pre-specified subgroup analyzes considered head position, age, sex, baseline neurological severity (NIHSS score), and medical history. To assist in understanding the adverse consequences of IUC use, we calculated a number needed to expose for poor outcome using the adjusted odds ratio (aOR) obtained in models. ${ }^{9}$ Data are reported with $95 \%$ confidence intervals $(\mathrm{CI})$, and a two-sided $\mathrm{P}<0.05$ was considered statistically significant. All analyses were performed with SAS version 9.3 (SAS Institute, Cary, NC).

\section{Data sharing}

Individual participant data used in these analyses can be shared by formal request with protocol from any qualified investigator to the Research Office of The George Institute for Global Health, Australia.

\section{Results}

Patient characteristics for IUC insertion

Of the 11,093 randomized HeadPoST patients (mean age 68 \pm 14 years; $60 \%$ male), 1167 (11.6\%) had an IUC inserted during their hospitalization, but this figure varied widely across regions: highest for participants in India/Sri Lanka (33.8\%), followed in decreasing frequency by those in South America, Australia/UK and China (including Taiwan) (Supplemental Table S3). The median duration over 7 days of IUC insertion was 5 days (IQR 3-7) (Supplemental Table S3).

Table 1 shows that the highest likelihood of IUC use was in older patients, and those with history of heart disease, greater initial neurological impairment, and a diagnosis of intracerebral hemorrhage (ICH). In particular, most (70.7\%) older patients ( $\geq 65$ years) had an IUC. Similarly, Day 7 data indicates that IUC use was related to greater neurological impairment and physical disability, defined by higher NIHSS and mRS scores, respectively. 
Moreover, IUC use was more frequent after an admission to an intensive care or acute stroke unit, and in those who received antibiotics within the 7 days, as compared to those without an IUC (Table 1).

\section{IUC and outcomes}

Median duration of IUC use in patients with a poor outcome (death or dependency, mRS 3-6) was significantly higher than in those with good functional recovery ( 6 vs. 5 days, $\mathrm{P}=0.013$; Supplemental Table S4). Compared to patients without an IUC, those with IUC had a greater likelihood of a poor outcome (76.6\% vs. 34.7\%; $\mathrm{P}<0.0001$; Supplemental Table S5). Table 2 shows that the increased odds of poor outcome with IUC use persisted after adjustment for imbalance in baseline characteristics and post-randomization management variables (Model III aOR 1.40, 95\%CI 1.13-1.74), and after multiple imputation of missing outcome data (aOR 1.36, 95\% CI 1.14-1.63). A significant interaction was found for IUC use and baseline NIHSS score (P for interaction 0.0019; Supplemental Figure S1). The number needed to be exposed to an IUC for harm (death or dependency) was 13.

Overall, only 0.7\% (76/11093) patients had a UTI (Supplemental Table S5), and this was more likely in those with IUC ( $1.5 \%$ vs. $0.6 \%, \mathrm{P}=0.0002$; Supplemental Table S5). The median time to diagnosis of UTI was 17 (IQR 5-36) days; but only 38\% (29/76) of UTI events occurred within Day 7/discharge (Supplemental Figure S2). However, in those patients with UTI, there was no clear difference in the median duration of IUC compared to those without UTI (4 [3-6] vs. 5 [3-7] days; P=0.17) (Supplemental Table S4). Table 3 shows this association in the initial adjusted analysis (model II, aOR 2.08, 95\% CI 1.13-3.82), but lost significance after adjustment for post-randomization management variables that included antibiotic use and Day 7 (or hospital discharge) neurological severity (model III, aOR $1.13,95 \%$ CI 0.59-2.18). These associations remained after multiple imputation (model 
III, OR 1.18, 95\% CI 0.63-2.19). In patients who received an IUC, duration of early use was not associated poor outcome (aOR 1.03, 95\% CI 0.93-1.14; Supplemental Table S6).

\section{Subgroup analysis}

The odds of poor outcome being higher in females ( $\mathrm{P}$ for interaction 0.01 ), the elderly (age >80 years; $\mathrm{P}$ for interaction 0.006 ), and those with mild neurological impairment (NIHSS scores of 0-4; P for interaction <0.0001) compared in other subgroups (Supplemental Figure S3). No heterogeneity was found for stroke subtypes ( $\mathrm{P}$ for interaction 0.47 ; Supplemental Figure S3), nor among subgroups for outcome in those with UTI (Supplemental Figure S4).

\section{Role of UTI on poor outcome}

Patients with UTI were more likely to have a poor 90-day outcome compared to those without UTI (71.9\% vs. 39.0\%; P<0.0001; Supplemental Table S2), but not after adjusting for all confounders (aOR 1.46, 95\% CI 0.70-3.04; Supplemental Table S7). The positive association of IUC and poor outcome persistent after including UTI as a confounder in the primary analysis (aOR 1.41, 95\% CI 1.13-1.75; Supplemental Table S7). Sensitivity analysis shows the positive association of IUC use and poor outcome was also present in patients without UTI, and of the same magnitude as identified in the primary analysis of the overall population (aOR 1.43, 95\% CI 1.15-1.78; Supplemental Table S8).

\section{Discussion}

In these secondary analyses of a large population of patients with acute stroke, we have shown that inserting an IUC was associated with a poor functional outcome after adjusting for various prognostic and management confounders, including a diagnosis of UTI and early in-hospital antibiotic use. 
Overall, approximately one in 10 stroke patients in our study had an IUC inserted, but the frequency was higher in the elderly and those with a history of heart disease, greater baseline neurological deficit, and ICH as the cause. As such, the lower overall frequency of IUC in our study than reported in registries $(20-30 \%)^{4,5,10}$ may reflect the selective nature of the clinical trial population which included patients with predominantly mild to moderate neurological severity, despite the pragmatic design and broad inclusion criteria. Yet, our data are consistent with others in showing an association of IUC with increasing age and stroke severity. ${ }^{4,11}$ Clearly, critical ill patients with more severe deficits are at increased risk of bladder dysfunction and adverse outcomes, and therefore, they require more intensive monitoring and interventional procedures, such as IUC insertion.

Explanations for the adverse consequences of IUC insertion not so clear cut. ${ }^{5}$ While an IUC may compromise early mobilization and rehabilitation, and cause an inflammatory reaction from subclinical urosepsis. ${ }^{12}$ Another explanation for the significant association of IUC and poor outcome is indication bias, whereby patients at risk of poor recovery (old age or severe disability) are more likely to receive an IUC as part of their management. Our subgroup analyses showed the adverse effect of IUC to be greater in patients with mild deficits (NIHSS scores 0-4) and early residual disability (mRS 0-2), suggesting caution in considering an IUC in such patients. Protocols outlining indications and management of IUC may help reduce complications and improve outcomes. ${ }^{13,14}$ Moreover, the finding elsewhere of females with poor functional stroke outcome ${ }^{15}$ having higher rates of inappropriate IUC use ${ }^{16}$ may explain our finding of sex differences in the prognostic significance of IUC.

A recent meta-analysis has shown a wide frequency (3-44\%) of UTI after stroke according to study design and setting. ${ }^{17}$ Once again, the low (0.7\%) frequency of UTI in HeadPoST compared to an observational study ${ }^{18}$ may relate to selection and observer bias, and diagnosis based on reported SAEs rather than systematic surveillance. However, our approach was 
arguably more clinically relevant in using a 'clinically significant' endpoint and in showing attenuation of the association of IUC use and UTI after adjustment for antibiotic use and level of functional impairment. Our finding are therefore contrary to a previous hospital-based study showing a significant association of IUC and UTI ${ }^{19}$ but these results were based on small sample where UTIs were reported without standard diagnostic criteria and there was no adjustment for other variables, such as antibiotic use. Our study also contrasts with another study which found an association of UTI and poor functional outcome (mRS 3-5) at the time of hospital discharge,${ }^{20}$ but is consistent with another study where adjustments were made for prognostic covariates. ${ }^{5}$ Nonetheless, we recognize that the small number of cases of UTI in our study restricted our ability to undertake stratified analyzes or adjust for a large number covariates.

Strengths of our study include the large sample size of participants with a broad range of characteristics managed in a range of health care settings. Moreover, we were able to adjust for a large number of potential confounding prognostic factors in different multivariable models. However, in any observational study there is the potential for incomplete adjustment, while the lack of systematic screening for UTI and reliance on SAE data, likely biased reported events towards those that were more clinically significant or symptomatic. We also had very limited information of the type of IUCs used, their indication, timing of removal, and relationship in the use of antibiotics. The data of censored assessment of all hospital management also limited the utility analysis of IUC duration and outcomes.

In summary, IUC use is associated with a poor functional outcome after acute stroke, after adjustment for a range of important prognostic and management factors. Further studies are required to establish causal pathways and inform guideline recommendations regarding the appropriate indications for IUC to decreases potential risks and promote recovery in vulnerable patients. 


\section{Author Contributions}

CSA and MO contributed to the concept and rationale for the study. MO undertook statistical analyses with assist from LB. MO wrote the first draft of manuscript with input from CSA. All authors commented upon and approved the final version of the manuscript for publication.

\section{Acknowledgements}

We thank the participants and investigators of the HeadPoST study.

\section{Disclosures}

PML reports grants from The George Institute for Global Health and Clínica Alemana de Santiago, during the conduct of the study; and non-financial support from Boehringer Ingelheim, grants and personal fees from Bayer and AstraZeneca, and grants from CONICYT, outside the submitted work. MLH holds a National Health and Medical Research Council of Australia (NHMRC) Career Development Fellowship. VVO reports grants from The George Institute for Global Health and Clínica Alemana de Santiago, during the conduct of the study; and research grants from Boehringer Ingelheim and CONICYT outside the submitted work. PMV reports grants from The George Institute for Global Health and Clínica Alemana de Santiago, during the conduct of the study; and research grants from CONICYT, outside the submitted work. SM was a member of the NHMRC Research Committee during 2015-2018. OMPN received grants for the Brazilian Stroke Research Network by DECIT/MS and CNPQ (402388/2013-5) for conduct this study. TGR is an National Institutes for Health Research (NIHR) Senior Investigator. CSA holds an NHMRC Senior Principal Research Fellowship, and reports honoraria and travel reimbursement from Takeda, Boehringer Ingelheim and Amgen outside of this study. The other authors have no disclosures to report. 


\section{References}

1. Kong KH, Young S. Incidence and outcome of poststroke urinary retention: A prospective study. Archives of physical medicine and rehabilitation. 2000;81:1464

2. Ersoz M, Tunc H, Akyuz M, Ozel S. Bladder storage and emptying disorder frequencies in hemorrhagic and ischemic stroke patients with bladder dysfunction. Cerebrovascular Diseases. 2005;20:395-399

3. Poisson NS, Johnston CS, Josephson AS. Urinary tract infections complicating stroke: Mechanisms, consequences, and possible solutions. Stroke. 2010;41:e180-e184

4. John G, Primmaz S, Crichton S, Wolfe C. Urinary incontinence and indwelling urinary catheters as predictors of death after new-onset stroke: A report of the south london stroke register. J. Stroke Cerebrovasc. Dis. 2018;27:118-124

5. Wu CH, Tseng MC, Chen YW, Sung SF, Yeh PS, Lin HJ. Indwelling urinary catheterization after acute stroke. Neurourology and Urodynamics. 2013;32:480-485

6. Powers JW, Rabinstein AA, Ackerson MT, Adeoye CO, Bambakidis MN, Becker CK, et al. 2018 guidelines for the early management of patients with acute ischemic stroke: A guideline for healthcare professionals from the american heart association/american stroke association. Stroke. 2018;49:e46-e99

7. Bowen A JM, Young, G. National clinical guideline for stroke prepared by the intercollegiate stroke working party. 2016

8. Anderson CS, Arima H, Lavados P, Billot L, Hackett ML, Olavarría VV, et al. Cluster-randomized, crossover trial of head positioning in acute stroke. The New England journal of medicine. 2017;376:2437

9. Bender R, Blettner M. Calculating the "number needed to be exposed" with adjustment for confounding variables in epidemiological studies. Journal of clinical epidemiology. 2002;55:525 
10. Net P, Karnycheff F, Vasse M, Bourdain F, Bonan B, Lapergue B. Urinary tract infection after acute stroke: Impact of indwelling urinary catheterization and assessment of catheter-use practices in french stroke centers. Rev. Neurol. 2018;174:145-149

11. Cowey E, Smith LN, Booth J, Weir CJ. Urinary catheterization in acute stroke: Clinical realities. A mixed methods study. Clinical Rehabilitation. 2012;26:470-479

12. Bernhardt BJ, English BC, Johnson BL, Cumming BT. Early mobilization after stroke: Early adoption but limited evidence. Stroke. 2015;46:1141-1146

13. Kim HJ, Chun MH, Han EY, Yi JH, Kim D-K. The utility of a bladder scan protocol using a portable ultrasonographic device in subacute stroke patients. Disability and Rehabilitation. 2012;34:486-490

14. Felix K. Decreasing catheter associated urinary tract infection in a long term acute care hospital. American Journal of Infection Control. 2016;44:S89

15. Mizrahi EH, Waitzman A, Arad M, Adunsky A. Gender and the functional outcome of elderly ischemic stroke patients. Archives of gerontology and geriatrics. 2012;55:438

16. Hu F-W, Chang C-M, Su P-F, Chen H-Y, Chen C-H. Gender differences in inappropriate use of urinary catheters among hospitalized older patients. Journal of Women \& Aging. 2019;31:165-175

17. Westendorp W, Nederkoorn P, Vermeij J-D, Dijkgraaf M, de Beek Dv. Post-stroke infection: A systematic review and meta-analysis. BMC Neurology. 2011;11:110

18. Stenzelius K, Laszlo L, Madeja M, Pessah-Rasmusson H, Grabe M. Catheterassociated urinary tract infections and other infections in patients hospitalized for acute stroke: A prospective cohort study of two different silicone catheters. Scandinavian Journal of Urology. 2016;50:483-488 
19. Stott DJ, Falconer A, Miller H, Tilston JC, Langhorne P. Urinary tract infection after stroke. QJM: An International Journal of Medicine. 2009;102:243-249

20. Popović N, Stefanović-Budimkić M, Mitrović N, Urošević A, Milošević B, Pelemiš M, et al. The frequency of poststroke infections and their impact on early stroke outcome. Journal of stroke and cerebrovascular diseases : the official journal of National Stroke Association. 2013;22:424 
Table 1. Characteristics of $\mathbf{1 1 0 9 3}$ patients by urinary catheter use

\begin{tabular}{|c|c|c|c|}
\hline \multirow[b]{2}{*}{ Characteristics } & \multicolumn{2}{|c|}{ Urinary catheter inserted } & \multirow[b]{2}{*}{$\mathrm{P}$ value* } \\
\hline & $\begin{array}{c}\text { Yes } \\
(\mathrm{N}=1167)\end{array}$ & $\begin{array}{c}\text { No } \\
(\mathrm{N}=9829)\end{array}$ & \\
\hline Age, years & $71.3 \pm 13.9$ & $67.5 \pm 13.7$ & $<0.0001$ \\
\hline Male & $610(52.3)$ & $5999(61.0)$ & $<0.0001$ \\
\hline Region & & & $<0.0001$ \\
\hline Australia/UK & $535(45.8)$ & $4154(42.3)$ & \\
\hline China (incl. Taiwan) & $266(22.8)$ & $4371(44.5)$ & \\
\hline India/Sri Lanka & $259(22.2)$ & $507(5.2)$ & \\
\hline South America & $107(9.2)$ & $797(8.1)$ & \\
\hline Hypertension & $630(54.0)$ & $4948(50.3)$ & 0.140 \\
\hline Previous stroke & $272(23.3)$ & $2314(23.5)$ & 0.854 \\
\hline Coronary artery disease & $192(16.5)$ & $1337(13.6)$ & 0.008 \\
\hline Atrial fibrillation & $227(19.5)$ & $936(9.5)$ & $<0.0001$ \\
\hline Heart failure & $70(6.0)$ & $337(3.4)$ & $<0.0001$ \\
\hline Diabetes mellitus & $252(21.6)$ & $1956(20.0)$ & 0.642 \\
\hline Stroke category & & & $<0.0001$ \\
\hline AIS & $950(81.5)$ & $8442(86.1)$ & \\
\hline $\mathrm{ICH}$ & $192(16.5)$ & $728(7.4)$ & \\
\hline Uncertain & $24(2.1)$ & $637(6.5)$ & \\
\hline NIHSS at admission & $12.0(6.0,18.0)$ & $4.0(2.0,7.0)$ & $<0.0001$ \\
\hline GCS score at admission & $14.0(11.0,15.0)$ & $15.0(14.0,15.0)$ & $<0.0001$ \\
\hline Pre-morbid mRS $0-1 \dagger$ & $881(75.5)$ & $7782(79.2)$ & 0.008 \\
\hline NIHSS at 7 days & $9.0(4.0,16.0)$ & $2.0(1.0,5.0)$ & $<0.0001$ \\
\hline $\mathrm{mRS}$ at 7 days & $4.0(3.0,5.0)$ & $2.0(1.0,3.0)$ & $<0.0001$ \\
\hline ICU admission & $242(20.7)$ & $274(2.8)$ & $<0.0001$ \\
\hline ASU admission & $824(70.6)$ & $5716(58.2)$ & $<0.0001$ \\
\hline Antibiotic treatment & $526(45.1)$ & $1146(11.7)$ & $<0.0001$ \\
\hline Underwent surgery & $29(2.5)$ & $7(0.1)$ & $<0.0001$ \\
\hline
\end{tabular}

Data are $\mathrm{n}(\%)$, mean (SD) and median (IQR).

AIS denotes acute ischaemic stroke, ASU acute stroke unit, GCS Glasgow coma scale, ICH intracerebral hemorrhage, ICU intensive care unit, mRS modified Rankin scale, NIHSS National Institutes of Health Stroke Scale.

*P values from the Student's t-test or Wilcoxon rank sum test for continuous variables, and the Chi-squared test or Fisher's extract test for categorical variables.

$\dagger$ Estimated functional grade score $0-1$ on the mRS

Includes decompressive hemicraniectomy, open craniotomy, minimally invasive surgery and intraventricular drainage of ICH. 
Table 2. Association of urinary catheter use and death or dependency at 90 days

\begin{tabular}{|c|c|c|c|c|}
\hline \multirow[t]{2}{*}{ Model } & \multicolumn{2}{|c|}{ Complete case dataset } & \multicolumn{2}{|c|}{ Multiple imputation dataset } \\
\hline & $\mathrm{aOR}(95 \% \mathrm{CI})$ & $\mathrm{P}$ value & $\mathrm{aOR}(95 \% \mathrm{CI})$ & $\mathrm{P}$ value \\
\hline Unadjusted* & $5.44(4.54-6.39)$ & $<0.0001$ & $5.17(4.44-6.02)$ & $<0.0001$ \\
\hline Model I $\uparrow$ & $5.27(4.49-6.18)$ & $<0.0001$ & $5.01(4.31-5.83)$ & $<0.0001$ \\
\hline Model II & $2.31(1.91-2.78)$ & $<0.0001$ & $2.21(1.85-2.64)$ & $<0.0001$ \\
\hline Model III§ & $1.40(1.13-1.74)$ & 0.002 & $1.36(1.14-1.63)$ & 0.001 \\
\hline
\end{tabular}

aOR denotes adjusted odds ratio, $\mathrm{CI}$ confidence interval

*Binomial logistic regression model with adjustment for the fixed effects of head position (lying-flat versus sitting-up) and crossover period, and random effects of cluster, and random interaction effects between cluster and crossover period.

$\dagger$ Model I adjusted for region (Australia/UK, China including Taiwan, India/Sri Lanka, South America)

$\$$ Model II is model I with further adjustment for baseline covariates of age as a continuous variable, sex, estimated premorbid grade 0 or 1 on the modified Rankin scale (mRS), baseline National Institutes of Health Stroke Scale (NIHSS) score as a continuous variable, history of heart disease (any heart failure, atrial fibrillation or coronary artery disease), diabetes mellitus or stroke, and pathological stroke type. $\S$ Model III is model II further adjusted for individual variables at Day 7 or earlier hospital separation, including intensive care unit (ICU) admission, acute stroke unit (ASU) admission, antibiotic use, and NIHSS score as a continuous variable. 
Table 3. Association of urinary catheter use and urinary tract infection within 90 days

\begin{tabular}{llllll}
\hline \multirow{2}{*}{ Model } & \multicolumn{2}{c}{ Original dataset } & & \multicolumn{2}{c}{ Multiple imputation } \\
\cline { 2 - 3 } \cline { 6 - 6 } & aOR $(95 \% \mathrm{CI})$ & P value & & aOR $(95 \% \mathrm{CI})$ & P value \\
\hline Unadjusted* & $2.85(1.62-4.99)$ & 0.0003 & & $2.85(1.62-4.99)$ & 0.0003 \\
Model I $\dagger$ & $2.58(1.47-4.53)$ & 0.001 & & $2.58(1.47-4.53)$ & 0.001 \\
Model II $\dagger$ & $2.08(1.13-3.82)$ & 0.018 & & $2.05(1.12-3.75)$ & 0.020 \\
Model III§ & $1.13(0.59-2.18)$ & 0.707 & & $1.18(0.63-2.19)$ & 0.610 \\
\hline
\end{tabular}

aOR denotes adjusted odds ratio, CI confidence interval

*Binomial logistic regression model with adjustment for the fixed effects of head position (lying-flat versus sitting-up) and crossover period, and random effects of cluster, and random interaction effects between cluster and crossover period.

$\dagger$ Model I adjusted for region (Australia/UK, China including Taiwan, India/Sri Lanka, and South America)

$\$$ Model II is model I with further adjustment for baseline covariates of age as a continuous variable, sex, estimated premorbid grade 0 or 1 on the modified Rankin scale (mRS), baseline National Institutes of Health Stroke Scale (NIHSS) score as a continuous variable, history of heart disease (any heart failure, atrial fibrillation or coronary artery disease), diabetes mellitus or stroke, and pathological stroke type.

§odel III is model II further adjusted for individual variables at Day 7 or earlier hospital separation, including intensive care unit (ICU) admission, acute stroke unit (ASU) admission, antibiotic use, and NIHSS score as a continuous variable. 